\title{
Spin-orbit coupling in graphene, silicene and germanene: dependence on the configuration of full hydrogenation and fluorination
}

\author{
RANBER SINGH \\ Department of Physics, Sri Guru Gobind Singh College, Sector 26, 160019 Chandigarh, India \\ ranber14@gmail.com
}

MS received 10 November 2017; accepted 1 August 2018; published online 5 December 2018

\begin{abstract}
We investigate the effect of full hydrogenation and fluorination on the spin-orbit coupling (SOC) in graphene, silicene and germanene. In chair conformation, the fluorination of graphene increases the spin-orbit splitting $\left(E_{\mathrm{so}}\right)$, while the hydrogenation and fluorination of other structures reduce the $E_{\mathrm{so}}$ at the $\Gamma$-point. In case of boat conformation, the hydrogenation and fluorination reduce the symmetry of honeycomb structure, which in turn remove the degeneracy of valence band maximum at the $\Gamma$-point. The change in band gaps due to SOC is very small in boat conformation structures as compared to that in the corresponding chair conformation structures.
\end{abstract}

Keywords. Halogenated graphene; spin-orbit interactions.

\section{Introduction}

The spin-orbit coupling (SOC) in a material is crucial for spin relaxation, spin-transport properties [1,2] and topological insulator states $[3,4]$. On one hand, the SOC has to be negligible for spin-relaxation and spin-transport applications $[1,2]$. On the other hand, the SOC has to be quite large to observe topological insulator states and hence, the quantum-spin hall effect (QSHE) states at realistic temperatures [3,4]. Ideal graphene and silicene have very small SOC, which is ideal for spin-transport applications. Functionalizing the graphene and related materials with adatoms or admolecules has emerged as very effective approach in these materials to tailor their electronic, spintronic and magnetic properties [5].

Graphene, silicene and germanene have zero band gap and linear energy dispersion near the Fermi level at the $\mathrm{K}$ and $\mathrm{K}^{\prime}$ points of the first Brillouin zone [6-9]. It means the energy spectrum at the $\mathrm{K}$ and $\mathrm{K}^{\prime}$ points is similar to the Dirac cones for the massless relativistic particles. The inclusion of SOC opens the band gap at the $\mathrm{K}$ and $\mathrm{K}^{\prime}$ points and change the ideal picture of observing relativistic particles in these materials $[3,10]$. However, the SOC opened band gap is very important for observing the QSHE states in these materials. Recently, the SOC in 2D graphene-like structures is extensively investigated [10-13] to open a significant band gap, so that the QSHE states can be realized in these structures at realistic experimental temperatures, which may be exploited for technological applications. The band gap opened due to SOC in planner honeycomb structures of graphene and silicene is very small, while it is about $2 \mathrm{meV}$ in germanene [9]. The buckling in these structures increase the SOC opened band gap significantly $[9,10]$. The role of buckling in the electronic structure of silicene and germanene structures was also investigated in detail by other research groups $[14,15]$.

The adatom defects are very effective in changing the properties of graphene and related 2D materials [5,11-13,16]. The adatoms induce the giant SOC locally in graphene [11-13]. The adatoms change the hybridization from $\mathrm{sp}^{2}$ to $\mathrm{sp}^{3}$ in the $\mathrm{C}$ atoms to which the adatoms are bonded. The heavy adatoms can also directly contribute to the SOC, because they have large intrinsic SOC. The change in SOC by the light adatoms, such as $\mathrm{H}$ is expected mainly due to the change in the hybridization from $\mathrm{sp}^{2}$ to $\mathrm{sp}^{3}$ in the $\mathrm{C}$ atoms at which the adatoms are bonded and to the local structural deformations.

In this paper, we investigate the effect of full hydrogenation and fluorination on the SOC in graphene $(\mathrm{C})$, silicene (Si) and germanene $(\mathrm{Ge})$. We consider the chair and boat conformation structures of hydrogenated $(\mathrm{HX}, \mathrm{X}=\mathrm{C}, \mathrm{Si}, \mathrm{Ge})$ and fluorinated (FX) material hexagonal honeycomb structure. Chair conformation has honeycomb structure, whereas boat conformation does not have honeycomb structure. We find that in the chair conformation structures, the hydrogenation and fluorination have significant effect on the SOC in graphene and germanene, while it has no significant effect on the SOC in silicene. The rest of the paper is organized as follows: in section 2, we present computational details, in section 3, the results are discussed in detail and finally, conclusions are drawn. 
Table 1. Calculated (LDA, PBE) and experimental (Exp.) values of lattice constant and spin-orbit splitting $\left(E_{\mathrm{so}}\right)$ at $\Gamma$ point in the diamond structures of $\mathrm{C}, \mathrm{Si}, \mathrm{Ge}$ and zinc-blende structure of cubic $\mathrm{SiC}$ (c-SiC). The calculated values of lattice constants and $E_{\mathrm{So}}$ with PBE pseudopotentials are in excellent agreement with the experimental data [21-23].

\begin{tabular}{lllllllrl}
\hline & \multicolumn{2}{c}{ Lattice constant $(\AA)$} & & \multicolumn{3}{c}{$E_{\text {so }}(\mathrm{meV})$} \\
\cline { 2 - 3 } \cline { 8 - 9 } Material & LDA & PBE & Exp. & & LDA & PBE & Exp. \\
\hline $\mathrm{C}$ & 3.53 & 3.57 & $3.57^{\mathrm{c}}$ & & 14.20 & 13.81 & $13^{\mathrm{a}}$ \\
$\mathrm{Si}$ & 5.39 & 5.47 & $5.43^{\mathrm{c}}$ & & 50.66 & 47.55 & $44^{\mathrm{b}}$ \\
$\mathrm{Ge}$ & 5.43 & 5.63 & $5.66^{\mathrm{c}}$ & & 323.10 & 293.66 & $290^{\mathrm{c}}$ \\
$\mathrm{c}-\mathrm{SiC}$ & 4.32 & 4.38 & $4.36^{\mathrm{c}}$ & & 14.98 & 14.54 & $10^{\mathrm{c}}$ \\
\hline
\end{tabular}

${ }^{\mathrm{a}}$ Ref. [21], ${ }^{\mathrm{b}}$ ref. [22], ${ }^{\mathrm{c}}$ ref. [23].

\section{Computational details}

The structural optimization and further electronic structure calculations are performed at the level of density functional theory (DFT) using Quantum-Espresso package [17] using ultrasoft pseudopotentials [18]. The exchange-correlation interactions are represented by using the local density approximation (LDA) [19] and the Perdew-Burke-Ernzerhof (PBE) type of generalized gradient approximation (GGA) [20]. The valence electron conformations used are $1 \mathrm{~s}^{1}$ for $\mathrm{H}, 2 \mathrm{~s}^{2} 2 \mathrm{p}^{2}$ for $\mathrm{C}$, and $2 \mathrm{~s}^{2} 2 \mathrm{p}^{5}$ for $\mathrm{F}, 3 \mathrm{~s}^{2} 3 \mathrm{p}^{2}$ for $\mathrm{Si}$ and $4 \mathrm{~s}^{2} 4 \mathrm{p}^{2}$ for Ge. The $\mathrm{k}$-points and a plane-wave basis set with a kinetic energy cutoff were optimized with respect to the total energy. We find $18 \times 18 \times 3 \mathrm{k}$-point mesh and a plane-wave basis set with a kinetic energy cutoff of $55 \mathrm{Ry}$ as the optimized parameters for

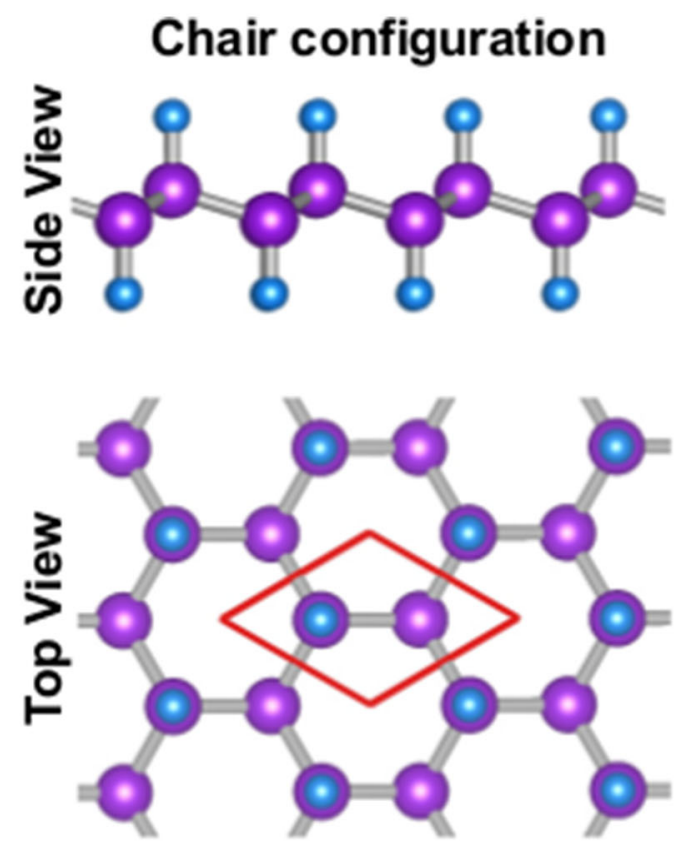

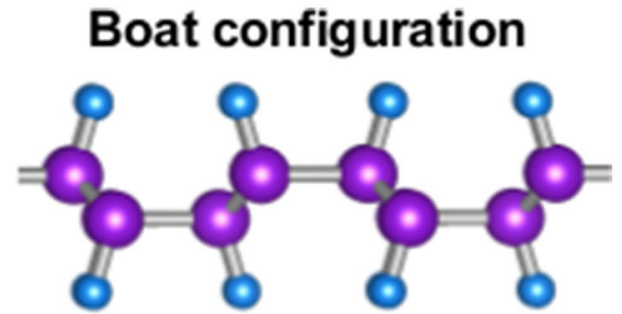

\section{Boat configuration}

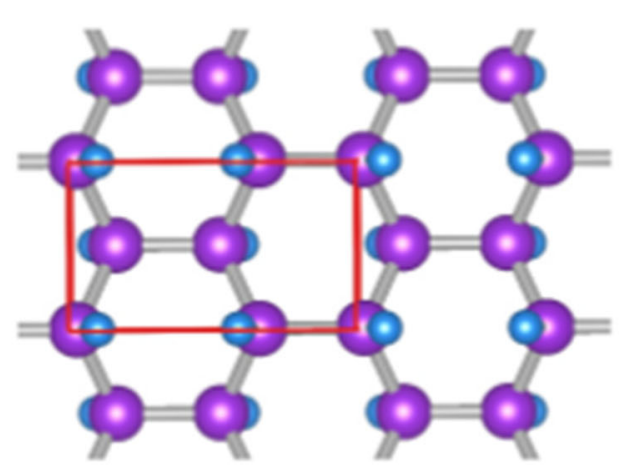

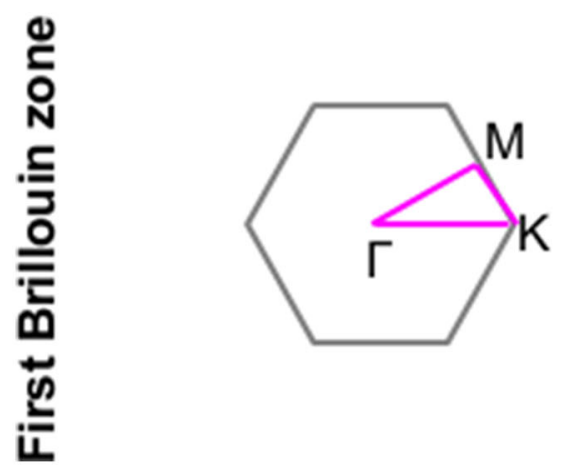

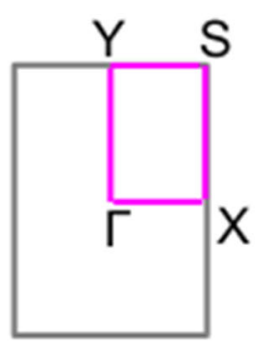

Figure 1. The chair and boat conformations of $2 \mathrm{D}$ hexagonal structures of $\mathrm{C}, \mathrm{Si}$ and $\mathrm{Ge}$ show the side and top views of the functionalized structures. The chair conformation structure has three-fold point symmetry (D3d $\overline{3})$, while the boat conformation structure has two-fold point symmetry (D2h-13). The red lines in the top views are marked to show the unit cells of these structures. In bottom, the corresponding first Brillouin zone structures are shown. 
the calculations with and without SOC. We used $18 \times 18 \times 3 \mathrm{k}$ point mesh for the structure optimization of hydrogenated and fluorinated graphene. A plane-wave basis set with a kinetic energy cutoff of $55 \mathrm{Ry}$ and density cutoff of $550 \mathrm{Ry}$ is used for representing the wave functions. The interactions between the adjacent sheets in the super cell geometry are hindered by a vacuum spacing of $12 \AA$.

We first optimize the crystalline structures and calculate the spin-orbit splitting at $\Gamma$-point of fcc Brillouin zone of 3D bulk structures of $\mathrm{C}, \mathrm{Si}, \mathrm{Ge}$ and cubic $\mathrm{SiC}$ using the LDA and PBE-GGA pseudopotentials. The convergence criteria of 0.00001 a.u. of the forces is used for the structure optimizations. The results are tabulated in table 1 . In the absence of $\mathrm{SOC}$, the valence band maximum (VBM) is three-fold degenerated. In the presence of SOC, the VBM splits into $J=3 / 2$ states and $J=1 / 2$ states. The energetic splitting between $J=3 / 2$ states and $J=1 / 2$ states is the quantity $E_{\text {so }}$ given in table 1. The results with PBE-GGA pseudopotentials are in excellent agreement with the available experimental data [21-23]. Thus, the level of DFT and pseudopotentials we use for our calculations is good enough to reproduce the experimental results. We then apply the same level of DFT, pseudopotentials and computational procedure to investigate the effect of full hydrogenation and fluorination on the SOC in 2D honeycomb structures of these materials.

The fully hydrogenated or fluorinated honeycomb structures of graphene exist in three stable conformations, namely; the chair, boat and washboard [24-28]. The chair conformation has four atoms, whereas boat and washboard conformations have eight atoms per unit cell. The chair conformation structure is lowest in energy [24,29]. The boat and washboard conformation structures have two-fold in-plane point symmetry, while chair conformation structure has three-fold in-plane point symmetry. We optimize fully hydrogenated and fluorinated structures of graphene, silicene and germanene in chair and boat conformations, which are lower in energy than washboard conformation structure. The chair conformation structure has three-fold $\mathrm{D} 3 \mathrm{~d} \overline{3}$ symmetry, while the boat conformation structure has two-fold D2h-13 symmetry. The spin-orbit splitting and the change in energy band gap after inclusion of SOC are investigated in these structures.

\section{Results and discussion}

Figure 1 shows the side and top views of chair and boat conformation structures of hydrogenated ( $\mathrm{HX} ; \mathrm{X}=\mathrm{C}, \mathrm{Si}, \mathrm{Ge}$ ) and fluorinated (FX) graphene $(\mathrm{C})$, silicene $(\mathrm{Si})$ and germanene (Ge). In chair conformation structure, the in-plane six-fold point symmetry of pure undecorated honeycomb structure is reduced to three-fold symmetry, while the boat conformation structure reduced point symmetry to two-fold. The corresponding first Brillouin zones structures are also shown in this figure. Chair conformation structure has hexagonal first
Table 2. Calculated values of lattice constants, $E_{\mathrm{so}}$ at the $\Gamma$ point, the band gaps $\left(E_{\mathrm{g}}\right)$ without spin-orbit coupling (WSOC) and change in band gap $\left(\Delta E_{\mathrm{g}}\right)$ with inclusion of SOC in the chair conformations of hydrogenated and fluorinated structures. The change in band gap is defined as $\Delta E_{\mathrm{g}}=E_{\mathrm{g}}$ (SOC) $-E_{\mathrm{g}}$ (WSOC). If the band gap without SOC is larger than band gap in the presence of $\mathrm{SOC}$, then, $\Delta E_{\mathrm{g}}$ is negative, otherwise, it is positive.

\begin{tabular}{lcrrr}
\hline Material & $\begin{array}{c}\text { Lattice } \\
\text { constant }(\AA)\end{array}$ & $E_{\text {so }}(\mathrm{meV})$ & $E_{\mathrm{g}}(\mathrm{eV})$ & $\Delta E_{\mathrm{g}}(\mathrm{meV})$ \\
\hline $\mathrm{C}$ & 2.461 & 9.519 & 0.000 & 0.001 \\
$\mathrm{HC}$ & 2.535 & 8.720 & 3.495 & -4.831 \\
$\mathrm{FC}$ & 2.601 & 27.113 & 3.144 & -13.929 \\
$\mathrm{Si}$ & 3.900 & 34.728 & 0.000 & 0.046 \\
$\mathrm{HSi}$ & 3.890 & 32.016 & 2.192 & -16.414 \\
$\mathrm{FSi}$ & 3.961 & 33.660 & 0.715 & -17.464 \\
$\mathrm{Ge}$ & 4.017 & 213.092 & 0.000 & 2.108 \\
$\mathrm{HGe}$ & 3.991 & 195.466 & 1.289 & -95.903 \\
$\mathrm{FGe}$ & 4.126 & 177.193 & 0.332 & -87.608 \\
\hline
\end{tabular}

Brillouin zone as that of pure pristine graphene, while boat conformation structure has rectangular first Brillouin zone.

The cubic diamond structure of $\mathrm{C}, \mathrm{Si}$ and Ge has threefold degenerated VBM at the $\Gamma$-point of the Brillouin zone. The inclusion of SOC partially removes this degeneracy and splits the VBM into $J=1 / 2$ (split-off band) and $J=3 / 2$ states (light-hole (LH) and heavy-hole (HH) bands) [21-23]. The HH and LH bands are still degenerated at the $\Gamma$-point in the presence of SOC. The calculated values of energetic difference $\left(E_{\mathrm{so}}\right)$ between split-off band and LH-HH band are in excellent agreement with available experimental data [21-23]. The HH and LH bands split under the influence of quantum confinement as in case of quantum structures such as quantum wells, quantum wires or quantum dots [30-32] and/or external fields such as strain [33], magnetic field [34], electric field [35], etc.

In 2D flat honeycomb structures of $\mathrm{C}, \mathrm{Si}$ and $\mathrm{Ge}$, the $\sigma \mathrm{VBM}$ at the $\Gamma$-point is two-fold degenerated, while the $\Pi$ bands near the $\mathrm{K}$ point are linear and has zero band gap. The inclusion of SOC completely removes this degeneracy of VBM at the $\Gamma$-point and opens a small gap at the K-point (table 2). This energetic splitting in VBM at $\Gamma$-point in the presence of SOC is the quantity $E_{\mathrm{so}}$ in $2 \mathrm{D}$ honeycomb structures given in table 2. The values of $E_{\mathrm{so}}$ in $2 \mathrm{D}$ honeycomb structures (calculated using PBE pseudopotentials) are significantly smaller than those in the corresponding 3D cubic diamond structures. The SOC band gap in 2D honeycomb structures is quite crucial for observing the QSHE states in these 2D structures. The SOC opened band gaps (with PBE pseudopotentials) are $0.001,0.046$ and $2.108 \mathrm{meV}$ in flat planner honeycomb structures of graphene, silicene and germanene, respectively (table 2). The SOC opened band gap between $\Pi$ bands at Kpoint is quite small as compared to the $E_{\mathrm{so}}$ of $\sigma \mathrm{VBM}$ bands at the $\Gamma$-point. This is due to the fact that the $\Pi$ electron hopping between two next nearest $\mathrm{C}$ atoms, which contributes 

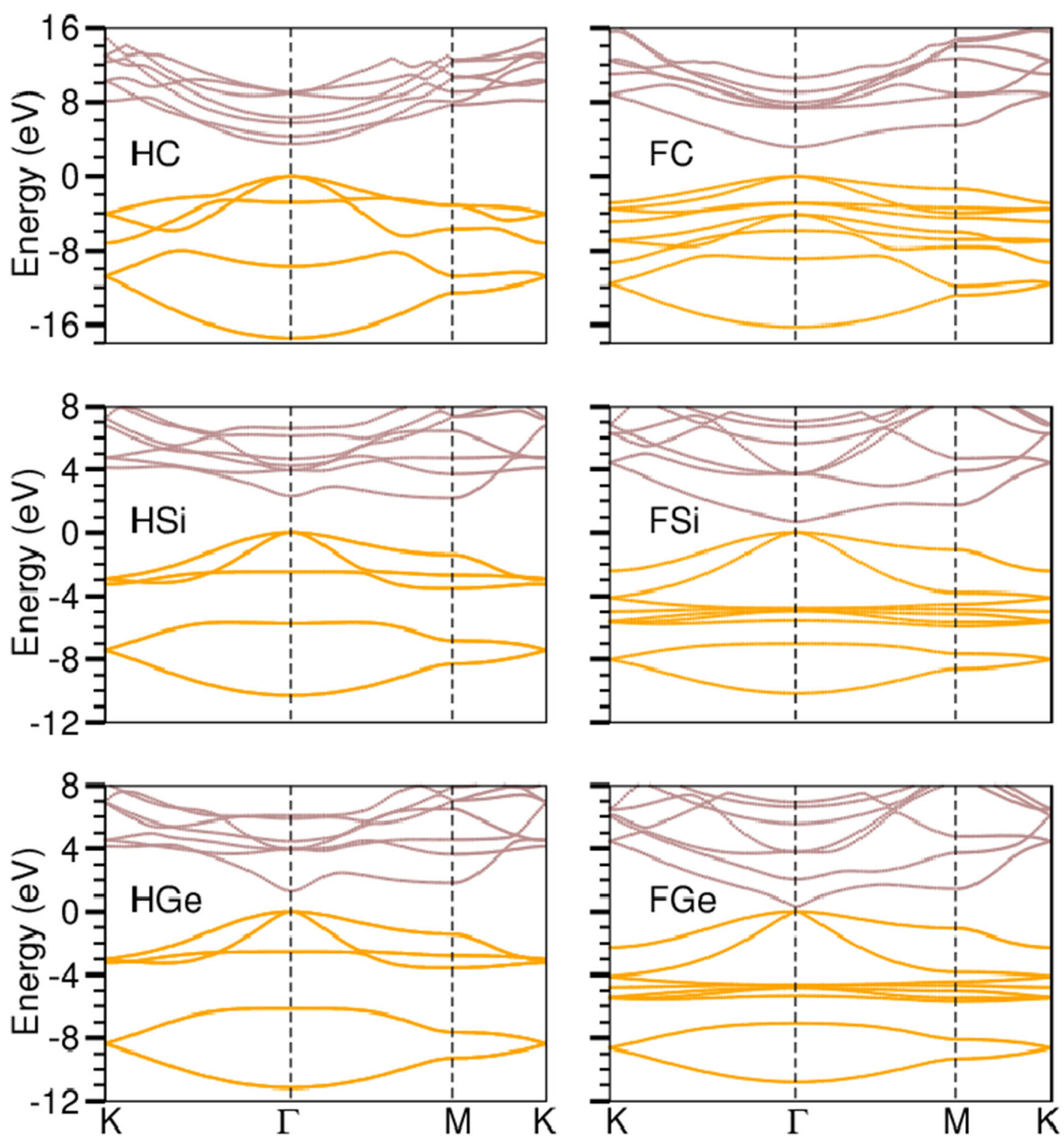

Figure 2. The electronic band structure of hydrogenated ( $\mathrm{HX} ; \mathrm{X}=\mathrm{C}, \mathrm{Si}, \mathrm{Ge})$ and fluorinated (FX) structures in the chair conformation. The orange (brown) lines indicate the filled (empty) electronic bands. The energy of VBM is set to zero in these plots. There is clearly noticeable difference between the CBM and VBM bands in hydrogenated structures and corresponding fluorinated structures. The HSi has indirect band gap with VBM at the $\Gamma$-point and CBM at the M point of first Brillouin zone, while all other structures have direct band gaps. The VBM has two-fold degeneracy at the $\Gamma$-point, which is removed with the inclusion of SOC. This energetic splitting in the VBM at the $\Gamma$-point in the presence of SOC is the quantity $E_{\text {so }}$ given in table 2.

to SOC, is a second-order process and comparatively quite weak [36,37]. The splitting at the $\Gamma$-point is caused by the $\sigma$ electron hopping, which is relatively stronger than $\Pi$ electron hopping.

The full hydrogenation or fluorination changes $\mathrm{sp}^{2}$ to $\mathrm{sp}^{3}$ hybridization of 2D honeycomb structures of $\mathrm{C}, \mathrm{Si}$ and $\mathrm{Ge}$. This removes the $\Pi$ bands in these structures. The fully hydrogenated and fluorinated structures have only $\sigma$ bands. In the chair conformation structure, the in-plane point symmetry is 3 -fold (D3d $\overline{3})$ as compared to six-fold point symmetry in honeycomb hexagonal structure of pure pristine graphene. However, in boat conformation, the point symmetry is reduced to two-fold point symmetry. Buckling heights of graphene, silicene and germanene after functionalization by $\mathrm{H}(\mathrm{F})$ atoms in chair conformations are $0.46(0.48), 1.95$ (1.98) and 197 (2.12) $\AA$, respectively.

Figure 2 shows the electronic band structure of hydrogenated and fluorinated chair conformations along the high symmetry directions of hexagonal first Brillouin zone. There is noticeable difference between the CBM and VBM bands 

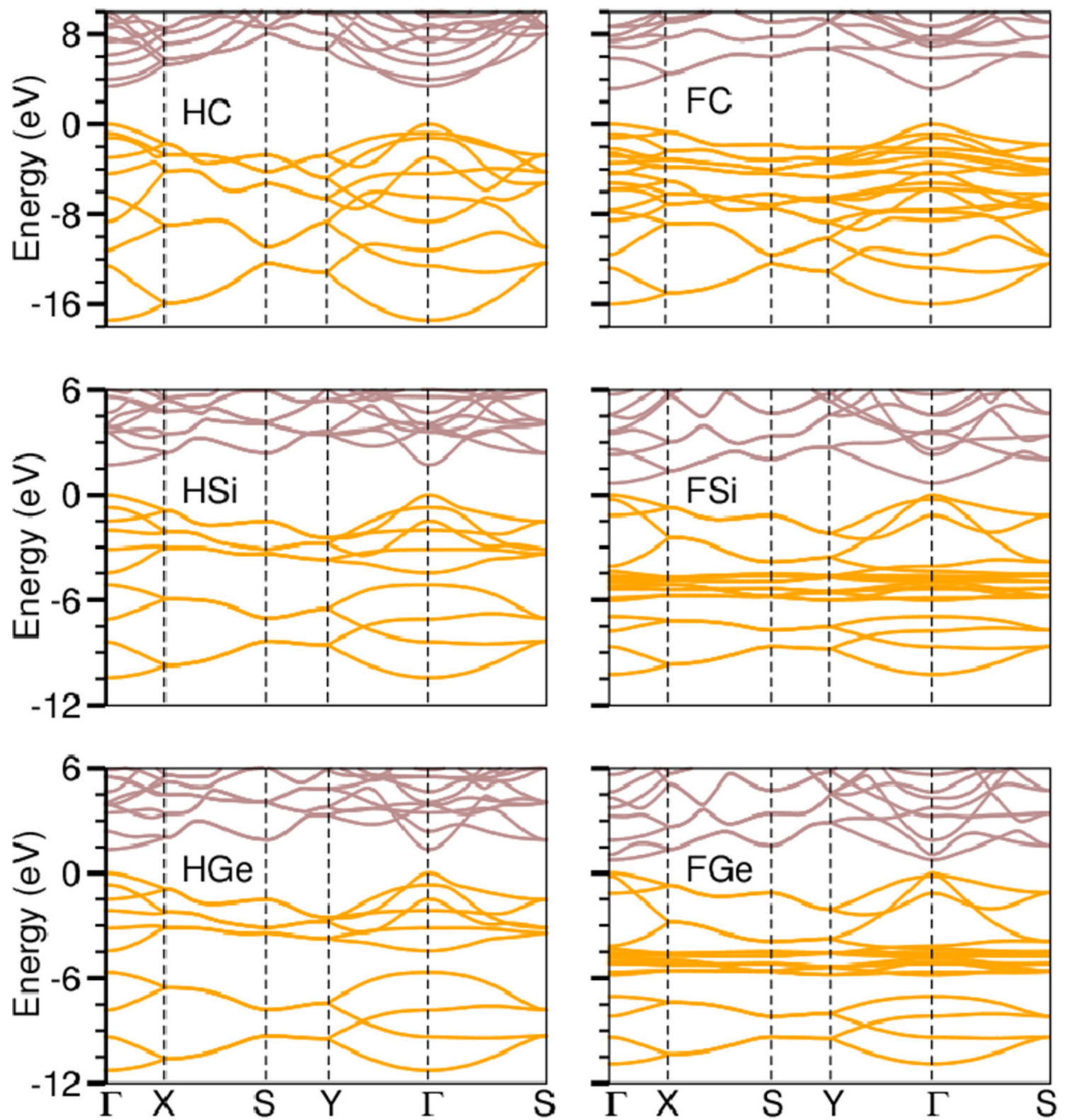

Figure 3. The electronic band structure of hydrogenated ( $\mathrm{HX} ; \mathrm{X}=\mathrm{C}, \mathrm{Si}, \mathrm{Ge})$ and fluorinated (FX) structures in the boat conformation. The orange (brown) lines indicate the filled (empty) electronic bands. The energy of VBM is set to zero in these plots. There is a noticeable difference between the CBM and VBM bands in hydrogenated structures and the corresponding fluorinated structures. All these structures have direct band gaps at the $\Gamma$-point of first Brillouin zone.

in hydrogenated structures and the corresponding fluorinated structures. The HSi has indirect band gap with VBM at $\Gamma$ point and CBM at $\mathrm{M}$ point of hexagonal first Brillouin zone, while all other structures have direct band gaps at the $\Gamma$-point. The VBM has two-fold degeneracy at the $\Gamma$-point similar to the case of $\sigma \mathrm{VBM}$ band in pristine honeycomb structures. The inclusion of SOC removes this degeneracy. The values of this energetic splitting $\left(E_{\mathrm{so}}\right)$ in VBM at $\Gamma$-point in the presence of SOC and change in band gap due to SOC are given in table 2.

Table 2 gives the values of lattice constants, $E_{\mathrm{g}}$ without SOC, $E_{\mathrm{so}}$ and $\Delta E_{\mathrm{g}}$ (the change in $E_{\mathrm{g}}$ ) with inclusion of SOC in pristine honeycomb structures and their fully decorated structures with $\mathrm{H}(\mathrm{F})$ in chair conformations. The change in band gap is defined as $\Delta E_{\mathrm{g}}=E_{\mathrm{g}}$ (SOC) $-E_{\mathrm{g}}$ (WSOC). If the band gap without $S O C$ is larger than the band gap in the presence of SOC, then $\Delta E_{\mathrm{g}}$ is negative, otherwise, it is positive. Thus, negative (positive) sign of $\Delta E_{\mathrm{g}}$ means the $E_{\mathrm{g}}$ decrease (increase) with inclusion of SOC. The inclusion of SOC decreases the band gap in hydrogenated and fluorinated chair conformations of graphene, silicene and germanene. However, in pristine graphene, silicene and germanene, the band gap at $\mathrm{K}$-point is increased with the 
inclusion of SOC. The SOC opened band gaps at K-point are $0.001,0.046$ and $2.108 \mathrm{meV}$ in graphene, silicene and germanene, respectively. The temperature equivalent of these band gaps are $0.011,0.53,24.5 \mathrm{~K}$, respectively. It means the QSHE states can be observed in germanene at quite realistic temperatures.

In case of graphene, the full fluorination increases $E_{\text {so }}$ significantly, while hydrogenation decreases it slightly. In case of silicene, there is small decrease in $E_{\text {so }}$ by hydrogenation and fluorination. In case of germanene, there is a significant decrease in $E_{\text {so }}$ by hydrogenation and fluorination. These different trends in different 2D materials are due to the contributions from the adatoms. In $\mathrm{HC}$, the contribution from $\mathrm{H}$ atoms is negligible, so that $E_{\text {so }}$ is almost same as in pristine graphene. In FC, there is significant contribution from $\mathrm{F}$ atoms, so that there is significant increase in $E_{\mathrm{so}}$, because intrinsic SOC of F atom is larger than that of $\mathrm{C}$ atom. This may also be due to local geometric distortions due to $\mathrm{F}$ adatoms. Nijamudheen et al [38] have also shown that depending upon the sites of $\mathrm{F}$ adatom addition, fluorinated graphene exhibit ferromagnetic/antiferromagnetic ground states. In HSi (FSi), there is negligible contribution from $\mathrm{H}(\mathrm{F})$ atoms, so that $E_{\text {so }}$ is almost same as in pristine silicene. In $\mathrm{HGe}$ (FGe), there is significant contribution from $\mathrm{H}(\mathrm{F})$ atoms, so that there is significant decrease in $E_{\mathrm{so}}$, because intrinsic SOC of $\mathrm{H}$ and $\mathrm{F}$ atoms is smaller than that of Ge atom. Due to larger splitting in the VBM at the $\Gamma$-point in the presence of SOC, there is larger band gap decrease in germanene as compared to graphene and silicene.

Figure 3 shows the electronic band structures of hydrogenated and fluorinated boat conformations along the high symmetry directions of rectangular first Brillouin zone. Similar to chair conformation structures, there is noticeable difference between the CBM and VBM bands in hydrogenated structures and the corresponding fluorinated structures. All of these structures have direct band gaps at the $\Gamma$-point. Since there are eight atoms per unit cell in boat conformation structures, therefore, these conformations have denser electronic band structures. Due to lower two-fold point symmetry in boat conformation structures, the VBM has no two-fold degeneracy at the $\Gamma$-point unlike in the case of honeycomb structures. The inclusion of SOC decreases the band gaps between CBM and VBM. The results of this effect of SOC on band gaps are given in table 3 .

Table 3 shows the optimized lattice constants, $E_{\mathrm{g}}$ without SOC and $\Delta E_{\mathrm{g}}$ with inclusion of SOC in the boat conformation structures of hydrogenated and fluorinated 2D structures of $\mathrm{C}, \mathrm{Si}$ and Ge. The inclusion of SOC decreases the band gap, but the change in band gaps is smaller as compared to that in the corresponding chair conformation structures. In the boat conformations of functionalized graphene and silicene by $\mathrm{H}$ or $\mathrm{F}$ atoms, the change in band gaps is very small, whereas in a boat conformation of functionalized germenene by $\mathrm{H}$ or $\mathrm{F}$ atoms, the change in band gap is quite significant. This is due to the increase in the hybridization of different bands by inclusion of SOC.
Table 3. Calculated values of lattice constants, the $E_{\mathrm{g}}$ without SOC and $\Delta E_{\mathrm{g}}$ with inclusion of SOC in the boat conformation of hydrogenated $(\mathrm{HX} ; \mathrm{X}=\mathrm{C}, \mathrm{Si}, \mathrm{Ge})$ and fluorinated (FX) structures. All these structures have direct band gaps at the $\Gamma$-point of the rectangular first Brillouin zone. Because of lower two-fold point symmetry of boat conformation structures, there is no degeneracy of VBM at the $\Gamma$-point. The inclusion of SOC affects VBM, but the change is not that large as in the case of chair conformation structures.

\begin{tabular}{|c|c|c|c|c|}
\hline \multirow[b]{2}{*}{ Material } & \multicolumn{2}{|c|}{ Lattice constant ( $\mathrm{A})$} & \multirow[b]{2}{*}{$E_{\mathrm{g}}(\mathrm{eV})$} & \multirow[b]{2}{*}{$\Delta E_{\mathrm{g}}(\mathrm{meV})$} \\
\hline & $a$ & $b$ & & \\
\hline $\mathrm{HC}$ & 4.303 & 2.523 & 3.387 & -0.470 \\
\hline $\mathrm{FC}$ & 4.600 & 2.574 & 3.170 & -0.605 \\
\hline $\mathrm{HSi}$ & 6.463 & 3.873 & 1.721 & -0.725 \\
\hline FSi & 6.693 & 3.959 & 0.641 & -1.797 \\
\hline $\mathrm{HGe}$ & 6.636 & 3.969 & 1.368 & -12.679 \\
\hline $\mathrm{FGe}$ & 6.944 & 4.117 & 0.768 & -38.006 \\
\hline
\end{tabular}

In summary, the SOC in hydrogenated and fluorinated structures of graphene, silicene and germanene is investigated using the ab-initio calculations based upon DFT and ultrasoft pseudopotentials. In chair conformations of hydrogenated $(\mathrm{HX} ; \mathrm{X}=\mathrm{C}, \mathrm{Si}, \mathrm{Ge})$ and fluorinated $(\mathrm{FX})$ structures, there is two-fold degeneracy of VBM at $\Gamma$-point. The inclusion of SOC removes this degeneracy at the $\Gamma$-point as in the case of pure honeycomb structure of $\mathrm{C}, \mathrm{Si}$ and $\mathrm{Ge}$. The fluorination of graphene increases the $E_{\mathrm{so}}$, while the hydrogenation and fluorination of other structures reduce the $E_{\text {so }}$ at the $\Gamma$-point. The band gaps in all the functionalized structures are reduced with the inclusion of SOC. In boat conformations of the functionalized structures, the symmetry of honeycomb structures is reduced and the degeneracy of VBM at $\Gamma$-point is removed. The changes in band gaps in boat conformations due to SOC are smaller as compared to that in the corresponding chair conformation structures.

\section{References}

[1] Tombros N, Jozsa C, Popinciuc M, Jonkman H T and van Wees B J 2007 Nature 448571

[2] Han W and Kawakami R K 2011 Phys. Rev. Lett. 107047207

[3] Kane C L and Mele E J 2005 Phys. Rev. Lett. 95226801

[4] Hasan M Z and Kane C L 2010 Rev. Mod. Phys. 823045

[5] Han W, Kawakami R K, Gmitra M and Fabian J 2014 Nat. Nanotech. 9794

[6] Castro Neto A H, Guinea F, Peres N M R, Novoselov K S and Geim A K 2009 Rev. Mod. Phys. 81109

[7] Cahangirov S, Topsakal M, Aktürk E, Sahil H and Ciraci S 2009 Phys. Rev. Lett. 102236804

[8] Houssa M, Scalise E, Sankaran K, Pourtois G, Afanas'ev V V and Stesmans A 2011 Appl. Phys. Lett. 98223107

[9] Singh R 2017 Int. J. Mod. Phys. B 321850055 
[10] Liu C-C, Feng W and Yao Y 2011 Phys. Rev. Lett. 107 076802

[11] Castro Neto A H and Guinea F 2009 Phys. Rev. Lett. 103 026804

[12] Weeks C, Hu J, Alicea J, Franz M and Wu R 2011 Phys. Rev. $X \mathbf{1} 021001$

[13] Gmitra M, Kochan D and Fabian J 2013 Phys. Rev. Lett. 110 246602

[14] Nijamudheen A, Bhattacharjee R, Choudhury S and Datta A 2015 J. Phys. Chem. C 1193802

[15] Jose D and Datta A 2014 Acc. Chem. Res. 47593

[16] Singh R and Kroll P 2009 J. Phys.: Condens. Matter 21196002

[17] Baroni S, de Gironcoli S, Dal Corso A and Giannozzi P 2001 Rev. Mod. Phys. 73515

[18] Vanderbilt D 1990 Phys. Rev. B 417892

[19] Perdew J P and Zunger A 1981 Phys. Rev. B 235048

[20] Perdew J P, Burke K and Ernzerhof M 1996 Phys. Rev. Lett. 77 3865

[21] Serrano J, Cardona M and Ruf T 2000 Solid State Commun. 113411

[22] Huldt L and Staflin T 1958 Phys. Rev. Lett. 1313

[23] Levinshtein M E, Rumyantsev S L and Shur M S 2001 (New York: Wiley) p 194

[24] Sofo J O, Chaudhari A S and Barber G D 2007 Phys. Rev. B 75 153401
[25] Park S and Ruoff R S 2009 Nat. Nanotechnol. 4217

[26] Elias D C, Nair R R, Mohiuddin T M G, Morozov S V, Blake P, Halsall M P et al 2009 Science $\mathbf{3 2 3} 610$

[27] Robinson J T, Burgess J S, Junkermeier C E, Badescu S C, Reinecke T L, Perkins F K et al 2010 NanoLett. 103001

[28] Nair R R, Ren W, Jalil R, Riaz I, Kravets V G, Britnell L et al 2010 Small 62877

[29] Singh R and Bester G 2011 Phys. Rev. B 84155427

[30] El Khalifi Y, Gil B, Mathieu H, Fukunaga T and Nakashima H 1989 Phys. Rev. B 3913533

[31] Csontos D, Brusheim P, Zülicke U and Xu H Q 2009 Phys. Rev. B 79155323

[32] Huo Y H, Witek B J, Kumar S, Cardenas J R, Zhang J X, Akopian N et al 2014 Nat. Phys. 1046

[33] Moise T S, Guido L J and Barker R C 1993 Phys. Rev. B 47 6758

[34] Kuhn-Heinrich B, Popp M, Ossau W, Bangert E, Waag A and Landwehr G 1993 Semi. Sci. Tech. 81239

[35] Miller D A B, Chemla D S, Damen T C, Gossard A C, Wiegmann W, Wood T H et al 1985 Phys. Rev. B 321043

[36] Min H, Hill J E, Sinitsyn N A, Sahu B R, Kleinman L and MacDonald A H 2006 Phys. Rev. B 74165310

[37] Yao Y, Ye F, Qi X-L, Zhang S-C and Fang Z 2007 Phys. Rev. B 75041401

[38] Nijamudheen A and Datta A 2013 J. Phys. Chem. A 1178506 